\title{
PARAMETRIC METHOD APPLICABLE IN CALCULATING BREAKOUT FORCE AND TIME FOR LIFTING AXISYMMETRIC OBJECTS FROM SEABED
}

\author{
Jan P. Michalski
}

Gdańsk University of Technology, Poland

\begin{abstract}
The issue of calculating parameters for lifting objects of axisymmetric shapes from the seabed is studied. The article presents the results of numerical simulation examination of the equation formulated by Foda for the force needed to extricate the object from the seabed depending on soil and water parameters, extrication speed, and object diameter. The simulations were performed within the range of parameters characteristic for real applications, and their results were used for deriving approximate algebraic formulas applicable in engineering practice of lifting objects resting on seabed.
\end{abstract}

Keywords: extricating objects from seabed; designing subsea recovery activities; predicting the breakout force

\section{INTRODUCTION}

The engineering practice in lifting objects from the seabed, such as a sunken shipwreck, a submarine, of a ballast anchor, for instance, reveals that the force needed to extricate the object from the seabed can be a multiple of its weight in water. The knowledge of this phenomenon and physical relations which describe it, along with the ability to predict the force, speed, and time needed for extricating the object from the seabed, can be a useful tool in both preparing the recovery procedure and selecting necessary instrumentation.

Empirical observations, quoted in [7] and [13] for instance, and experimental tests, such as those described in [2] and [3], give the evidence that the phenomenon of object sucking to the seabed results from local pressure differences generated in water and in the porous seabed soil when the object is extricated from the seabed. The global effect of this phenomenon is the increasing sucking force when the object is moved away from the seabed, along with the resulting increase of the force needed to extricate it, above its static weight in water. Correct force assessment is essential when lifting such objects as ballasts, dead anchors, tanks resting on the seabed, construction plates, underwater pipelines, or shipwrecks. A wide spectrum of the quoted examples testifies that the issue of object extrication from seabed is of high importance in widely understood ocean engineering activities.

The literature review on the subject reveals that the value of the extrication resistance force is related in a complicated way with the time and speed of object extrication, and with hydraulic and mechanical properties of the seabed soil and water in it. One of main difficulties when conducting experimental investigations of the above phenomenon is that its real physical and geometric conditions cannot be precisely modelled in the laboratory scale. As a consequence, the results of laboratory experiments cannot be easily extrapolated to real conditions, the more so that the laws of similitude for this phenomenon have not been well-defined [12] and there is a need to refer to the theoretical knowledge and analytical methods. A conceptual sketch of the studied issue is given in Fig. 1. 


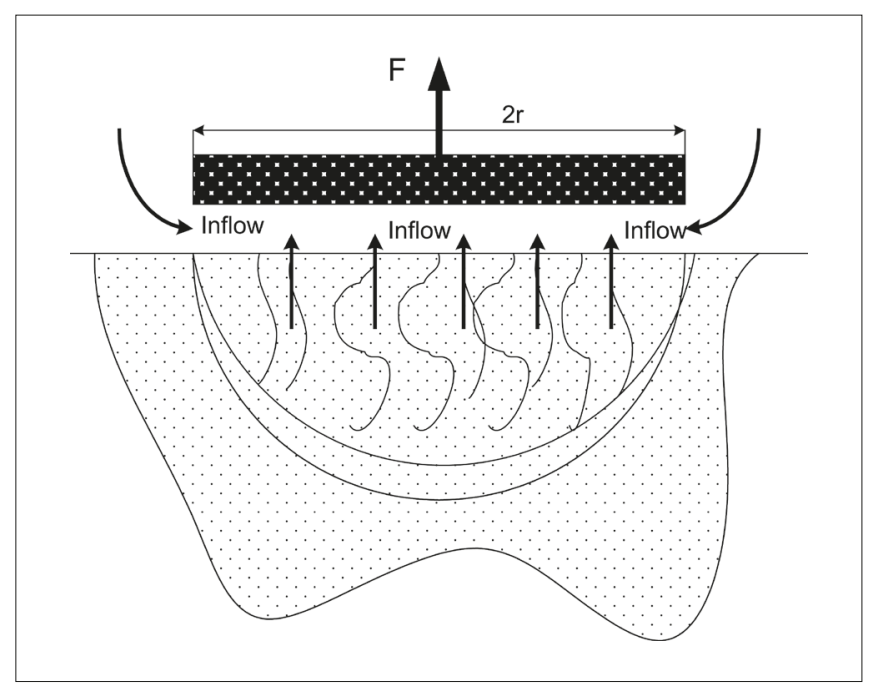

Fig. 1. Conceptual sketch of the phenomenon of object extrication from porous seabed

\section{PURPOSE AND SCOPE}

The well-known methods to determine forces needed to lift an object resting on the seabed are either simple, with low assessment accuracy resulting from neglecting a number of important parameters of the phenomenon (for instance, the formula given by Poinc [10]), or take into account many essentials at the expense of high complication of mathematical description and difficulties in calculating parameters important for engineering practice.

The purpose of the study reported in this article was developing a method to determine essential parameters for the technology of object lifting from seabed, which would have simple mathematical structure and the accuracy comparable with that of advanced theoretical methods, thus being applicable in engineering practice.

However, the applicability of the developed method has some limitations, as its range comprises only basic extrication parameters, such as the shape and dimensions of the lifted object, the extrication force, speed, and time, the total time of the breakout phenomenon, and the time after which the sucking action of the seabed on the object disappears. The motivation and inspiration for undertaking this task was the research done within the framework of the previous project [9]. An attempt to use the Foda model for practical calculations was presented in [12], while more systematic studies were conducted by Liu [6]. However, the results of these studies were only described in an internal report and are not widely available.

\section{LITERATURE REVIEW ON THE SUBJECT}

The force needed for extricating a body from the seabed can be roughly assessed using non-structural approximate empirical relations, such as the formula proposed by Poinc [10], which says how much the force needed to extricate the object from the seabed is larger than the weight of the object in water. This force/weight ratio is calculated taking into account the type of seabed, starting from stony and ending with clay type. In the Poinc formula, the suction force only depends on the type of base soil, which is a major simplification, as both practical experience and performed experiments show that the extrication force, speed, and time are mutually dependent.

It results from the laboratory tests performed by Basinski [2], [3] that the extrication resistance force, also referred to as the suction force, can reach a large value, which is also confirmed by reports from excavation works cited both by the author and in [14]. Basinski's motivations for undertaking labour-intensive experimental investigations was that the available literature contains insufficient amounts of model test data for reliable generalisation concerning their scaling to real objects.

In the tests performed by Basinski, the dimensions of the extricated object are large (up to $30 \mathrm{~cm}$ ), compared to the dimensions of soil particles. In the light of his research, the course of object extrication from granular soil during the phase of suction force action is as follows. The applied external lifting force reduces the pressure under the rigid body base with respect to the surrounding hydrostatic pressure. The reduced pressure distribution under the object base changes when the object rests freely on the soil surface, and is uniform when the object is embedded into the soil. The pressure reduction generated by the object motion propagates into the soil, and the appearing pressure gradient initiates the flow of the water filling the soil structure (pores).

At object edges, the pressure difference makes that the water flows faster under the object base, as a result of which the soil becomes more liquefied and is washed out. The water flow generates relatively large forces of inertia of water masses and friction forces between water particles moving up and soil particles, all this leading to the reduction of soil cohesion and lifting up of part of soil particles. With the increasing gap between the rigid body base and the soil, the motion resistance in the gap decreases and the amount of water flowing through it increases.

Theoretical analyses of the above issue are based on the concept of boundary layer in porous medium. It is assumed that in direct vicinity of the seabed surface, the dynamics of motion of the water filling the soil pores differs from that inside the soil medium. This assumption is justified by the fact that the value of the suction force depends on the speed of extricating the object from the seabed, as well as on the type and cohesion of the soil, the air content in the water, the geometry and dimensions of the object, and on how deep the object is embedded into the soil.

Based on the varying pressure distribution generated by the force used to extricate the object from the seabed, three phases of the phenomenon can be named. In the first phase, which lasts until the stability of the soil structure skeleton is lost, the breakout force is mainly composed of adhesive and cohesive forces acting on the contact surface between the extricated body and the soil. In the second phase, starting after the soil stability is lost, the dominating force is the resistance of the water flowing through the soil, while in the 
third phase - the resistance of the water flowing through the gap between the object and the seabed. This scheme provides an opportunity for extracting the static breakout resistance, acting until the time of clear beginning of motion, and the kinetic extrication resistance, acting in the phase of object motion.

Sawicki [13] considers the issue in which the lifted object moves uniformly and rectilinearly in the direction of action of gravity forces. The structure of the water reservoir bottom is modelled as the porous body filled with two-phase compressible liquid. The compressibility of the liquid is described by the parameter $\beta$, the value of which depends on water aeration in pores. The elasticity of the porous medium structure is assumed and defined by the parameter $G$. This medium property plays a vital role in modelling flows in pores during rapid appearance of negative pressure (suction). The third important parameter is the seabed porosity coefficient $n$, the values of which do not differ much in the examples analysed in the literature.

An interesting mathematical model of axisymmetric object extrication from the seabed is proposed by Foda [4], who makes a series of simplifying assumptions to derive a formula describing the pressure decrease between the seabed structure and the adhering object at the time of object motion initiation. Making use of Biot's theory about properties of flows in porous-elastic bodies and assuming that the flow in pores obeys Darcy's law, the author derives formulas describing the dynamic pressure distribution and the resulting force of object suction to the bottom of water reservoir.

For the adopted assumptions, Foda obtained formulas being the solutions for axisymmetric objects (with respect to vertical axis) and slender objects. The advantage of the Foda method in the aspect of practical applications is its prognostic value - the delivered solutions include the phenomenon referred to as "breakout", well-known from practice, which consists in the appearance of maximal breaking force after some time from the beginning of object extrication from the seabed, followed by fast decrease of the sucking force. A detailed analysis of assumptions made and results obtained by Foda [4] was done by Mei et al. [7] who stressed the importance of the use of different models of soil structure depending on its type.

\section{CONCEPT AND RESULTS OF THE STUDY}

The prognostic quality of the Foda method [4] justifies its use for systematic simulation studies covering a wide range of practically used parameter values and expected to provide data applicable for deriving simple and useful approximation formulas, without need to solve complicated equations such as Eq. (1) or Eq. (1).

According to Foda [4], for the motion with nonuniform extrication speed, the force $F$ with which the axisymmetric object is sucked to the seabed is given by Abel's differentialintegral equation:

$$
F(t)=\pi \cdot r^{2} \cdot \alpha \cdot \int_{0}^{t}\left[1-\frac{2 \cdot I_{1}\left(f_{o} \cdot r\right)}{f_{o} \cdot r \cdot I_{0}\left(f_{o} \cdot r\right)}\right] \cdot\left(\frac{d \Delta}{d \tau}\right) \frac{d \tau}{(t-\tau)^{1 / 2}}
$$

When the extrication speed $w$ is uniform, the extrication force is given by the integral equation:

$$
F(t)=\pi \cdot r^{2} \cdot \alpha \cdot w \cdot \int_{0}^{t}\left[1-\frac{2 \cdot I_{1}\left(f_{o} \cdot r\right)}{f_{0} \cdot r \cdot I_{0}\left(f_{0} \cdot r\right)}\right] \cdot \frac{d \tau}{(t-\tau)^{1 / 2}}
$$

The symbols in the above equations have the following

$$
\begin{aligned}
& \alpha=\frac{1+m}{m} \cdot\left[\frac{\frac{G}{k}}{\pi \cdot m \cdot(1-2 \cdot v)+\frac{\pi(1-2 \cdot v)}{2 \cdot(1-v)}}\right]^{\frac{1}{2}} \\
& f_{o}=\left(\frac{6 \cdot \mu}{\alpha \cdot w^{3}}\right)^{\frac{1}{2}} \cdot t^{-\frac{7}{4}} \quad m=\frac{n \cdot G}{(1-2 \cdot v) \cdot \beta} \quad k=\frac{\beta}{\gamma}
\end{aligned}
$$

Names and values of parameters used in the simulation studies are as follows:

$t$

$w \quad-$ extrication speed;

$r \quad-$ radius of the object;

$I_{0}() \quad-$. zero-order modified Bessel function of first kind;

$I_{1}() \quad-$. first-order modified Bessel function of first kind;

$\Delta \quad-$ object displacement distance;

$G=10^{7} \mathrm{~N} / \mathrm{m}^{2} \quad-$ shear modulus of seabed solid;

$\beta=10^{7} \mathrm{~N} / \mathrm{m}^{2} \quad$ - bulk modulus of porous fluid (partial saturation);

$k=10^{-10} \mathrm{~m}^{3} \mathrm{~s} / \mathrm{kg}$ - soil permeability coefficient;

$n=0.3 \quad$ - seabed porosity coefficient;

$\mu=1.325 \mathrm{t} /(\mathrm{m} \cdot \mathrm{s}) \quad$ - water viscosity coefficient;

$\gamma=10^{4} \mathrm{~N} / \mathrm{m}^{3} \quad-$ specific weight of water;

$m=0.9 \quad-$ ratio of solid skeleton elasticity to fluid elasticity;

$v=0.333 \quad-$ Poisson's ratio of solid-fluid system;

The following ranges of parameters were considered interesting from the point of view of engineering practice and used in the performed simulations:

- set of object radii:

$$
r \in\{1.00 \mathrm{~m}, 5.00 \mathrm{~m}, 10.00 \mathrm{~m}\}
$$

- set of uniform extrication speeds:

$w \in\left\{10^{-3} \mathrm{~m} / \mathrm{s}, 10^{-4} \mathrm{~m} / \mathrm{s}, 10^{-5} \mathrm{~m} / \mathrm{s}, 10^{-6} \mathrm{~m} / \mathrm{s}, 10^{-7} \mathrm{~m} / \mathrm{s}\right\} ;$

- limiting value of extrication force decay $F_{\text {decay }}=10 \mathrm{~N}$.

At low extrication speeds, solving numerically Eq. (2) becomes difficult, as in this case the argument $f_{o}$ in Eq. (4) takes high values, which exceed the applicability range of 
classical formulas for calculating Bessel functions. In this case, use was made of asymptotic formulas given in [1], [5], [8], [11]. After some preliminary tests, the following approximation was selected for simulation calculations:

$$
\frac{I_{1}(x)}{I_{0}(x)} \cong \exp \left(\frac{-1}{2 \cdot x}\right) \text { for } x>800 \text { with }: O\left(\frac{-1}{x^{2}}\right)
$$

The extrication forces and gap dimensions calculated from Eq. $f_{0}$ are shown in Fig. 2, Fig. 3 and Fig. 4, for different object dimensions. In these figures, the logarithmic scale was used, as the values of the presented parameters differ by several orders of magnitude. Continuous lines are graphical representations of solutions of Eq. (2) with respect to the extrication force, while broken lines illustrate the size of the gap between the seabed and the object, depending on the extrication speed and time.

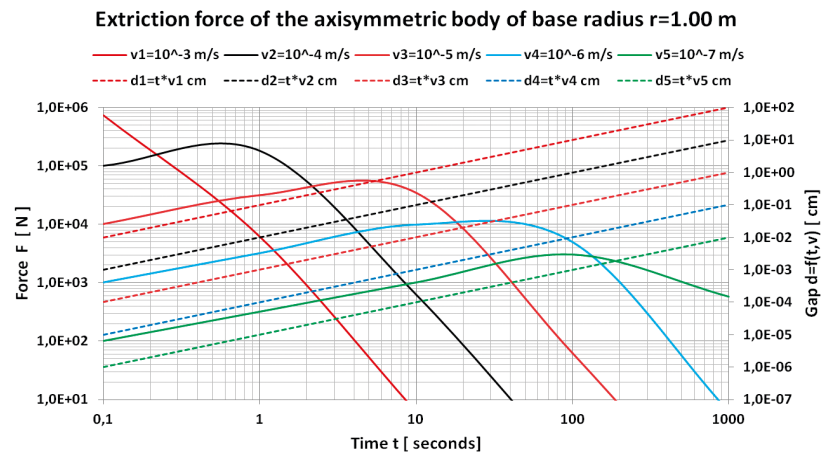

Fig. 2. Time-histories of extrication force and gap size at different extrication speeds; $r=1 \mathrm{~m}$

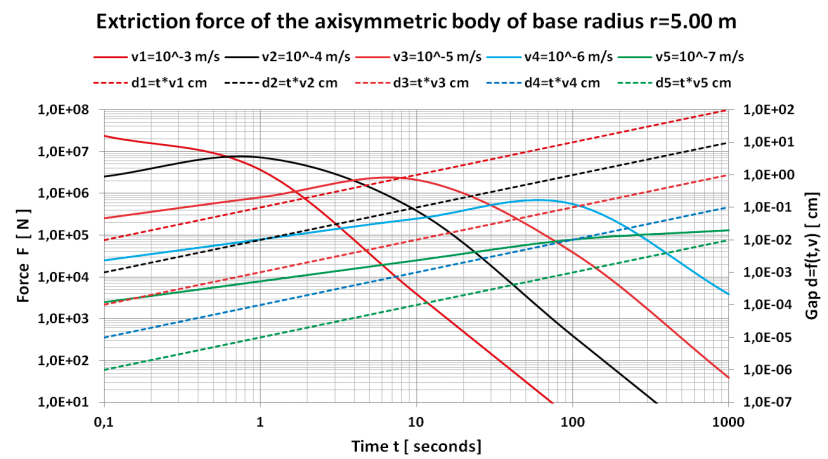

Fig. 3. Time-histories of extrication force and gap size at different extrication speeds: $r=5 \mathrm{~m}$

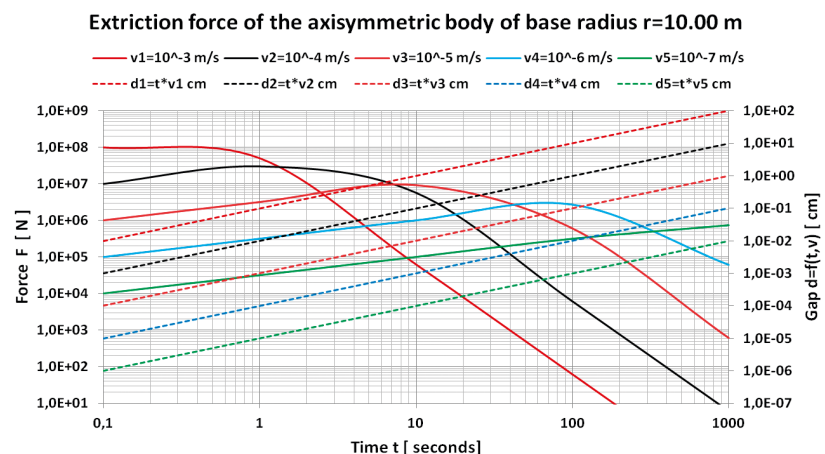

Fig. 4. Time-histories of extrication force and gap size at different extrication speeds: $r=10 \mathrm{~m}$
The maximal extrication force $F_{\text {max }}$, often referred to as the "breakout force", and the time $T_{\max }$ after which this force appears were calculated, for different object sizes and extraction speeds, from the precondition for the existence of extremum of suction force characteristic:

$\frac{d F(t)}{d t}=\pi \cdot r^{2} \cdot \alpha \cdot w \cdot \frac{d}{d t} \int_{0}^{t}\left[1-\frac{2 \cdot I_{1}\left(f_{o} \cdot r\right)}{f_{0} \cdot r \cdot I_{0}\left(f_{o} \cdot r\right)}\right] \cdot \frac{d \tau}{(t-\tau)^{1 / 2}}=0$

The maximum forces $F_{\max }$ calculated from Eq. (6) are shown in Fig. 5 as continuous lines.

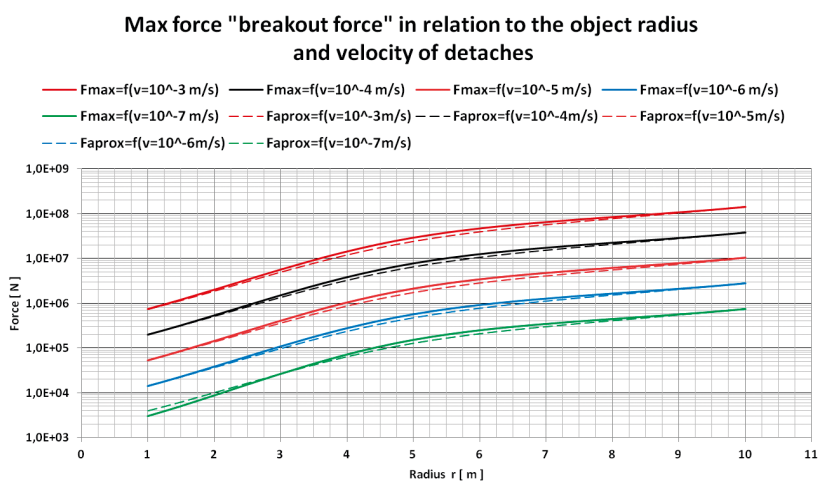

Fig. 5. Maximal extrication forces (breakout forces) as functions of object size and extrication speed

An interesting parameter of the study is the time $T_{\text {decay }}$, after which the sucking action of the seabed on the extricated object decays. It was assumed that the decrease of the sucking force below $F_{\text {decay }}=10 \mathrm{~N}$ means the decay of the suction effect of seabed, after which the further process of object lifting becomes a classic task of object moving in water, where the resistance force depends on the shape and weight of the object and the speed of its lifting.

The time $T_{\text {decay }}$ was determined by solving the following equation for time $t$

$$
F_{\text {decay }}-\pi \cdot r^{2} \cdot \alpha \cdot w \cdot \int_{0}^{t}\left[1-\frac{2 \cdot I_{1}\left(f_{o} \cdot r\right)}{f_{o} \cdot r \cdot I_{0}\left(f_{o} \cdot r\right)}\right] \cdot \frac{d \tau}{(t-\tau)^{1 / 2}}=0
$$

The calculated values of $T_{\max }$ after which the maximum forces occur are shown in Fig. 6 as continuous lines, while the time $T_{\text {decay }}$ after which the sucking effect of seabed decays are represented by broken lines.

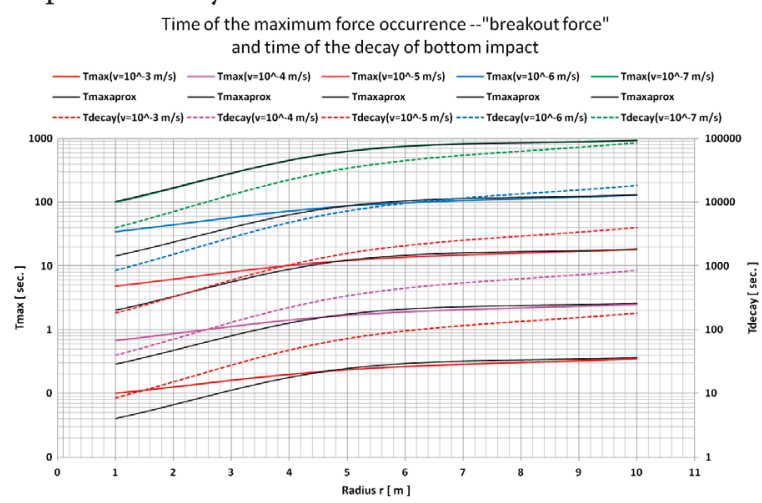

Fig. 6. Time $T_{\max }$ of the occurrence of breakout force $F_{\max }$ and time $T_{\text {decay }}$ of suction force decay 
The results of simulation calculations comprise discrete characteristics which represent relevant relations for fixed values of parameters $r_{i}$ and $w_{i}$ :

$$
\begin{aligned}
& -F_{\text {max } i}=f\left(r_{i}, w_{i}\right) ; \\
& -T_{\text {max } i}=f\left(r_{i}, w_{i}\right) ; \\
& -T_{\text {decay } i}=f\left(r_{i}, w_{i}\right) .
\end{aligned}
$$

The sets of discrete values of these characteristics were used for deriving simple and continuous algebraic formulas which relatively precisely approximate the original discrete characteristics. The adopted measure of accuracy of the obtained approximations was the square of the relative mean error. From among the tested hypothetical formulas approximating the maximum force characteristics, the best approximation was given by the formula:

$$
\operatorname{approx} F_{\max }=94.85 \cdot w^{0.569} \cdot\left(r^{0.7364}+0.663\right)^{4.048}
$$

In Eqs. (8), (9) and (10), the extrication speed $w$ is expressed in $[\mathrm{mm} / \mathrm{s}]$, the object radius $r$ in $[\mathrm{m}]$, and time in $[\mathrm{s}]$. approx $F_{\text {max }}$ is the maximum force value expressed in $[\mathrm{kN}]$.

The values of the force approx $F_{\text {max }}$ are shown in Fig. 5 as broken lines, which provides opportunities for comparing them with original values (continuous lines) for different extrication speeds and different object sizes. The force approx $F_{\max }$ only represents the suction force, which means that it should be enlarged by the weight of the object in water when calculating parameters for the lifting device.

A similar approach was used when deriving the approximating formulas approx $T_{\max }$ for the time after which the maximum force occurs, and approx $T_{\text {decay }}$ for the time after which the sucking effect of seabed decays. For the former case, the best approximation had the form:

$$
\operatorname{approx} T_{\max }=0.101 \cdot w^{-0.852} \cdot\left(r^{1.96}-0.96\right)^{0.286}
$$

In Fig. 6, coloured continuous lines represent the original solutions, while thin black lines present the relations given by Eq. (9).

For the time approx $T_{\text {decay }}$ after which the action of the sucking force decays, the best approximation was:

$$
\text { approx } T_{\text {decay }}=8.55 \cdot w^{-2 / 3} \cdot\left(r^{0.973}-0.00028\right)^{1.37}
$$

In Fig. 6, coloured broken lines represent the relations given by Eq.(10). In Eq. (9) and Eq. (10) the speed $w$ is expressed in $[\mathrm{mm} / \mathrm{s}]$, the object radius in $[\mathrm{m}]$, and time in $[\mathrm{s}]$.

\section{SAMPLE METHOD APPLICATION}

The extrication speed and time are to be assessed for a circular object of $r=2.40 \mathrm{~m}$, assuming that the available lifting force is $350 \mathrm{kN}$.
Placing the force and object radius values into Eq. (8) and performing some algebraic operations leads to the relation: $0.08104=w^{0.569}$. Raising both sides of the equation to a power of $\frac{1}{0.569} \cong 1.757$ gives the speed $w=0.08104^{1.757} \cong 0.012 \mathrm{~mm} / \mathrm{s}$. After placing the speed and object radius values into Eq. (9) we get the time after which the maximum force occurs, $T_{\text {max }} \cong 6.7 \mathrm{~s}$, and the gap size at this time, $d_{\text {max }} \cong 0.08 \mathrm{~mm}$. Finally, placing the same values into Eq. (10) gives us the time after which the suction force decays, $T_{\text {decay }} \cong 521 \mathrm{~s}$, and the corresponding gap size, $d_{\text {decay }} \cong 6.2 \mathrm{~mm}$.

The presented results of the study can make a basis for developing a programme of laboratory experiments, while practical applicability of the proposed method consists in fact that the above calculations can be performed using a simple calculator.

\section{REFERENCES}

1. Amos D. E.: Computation of Modified Bessel Functions and Their Ratios. Mathematics of Computation Vol. 28, No. 125 (Jan., 1974).

2. Basiński T.: Resistance force during extricating a rigid body from sandy bottom of water reservoir (in Polish). Archiwum Hydrotechniki. Vol. XVI, No. 4, 1969.

3. Basiński T.: Laboratory tests of resistance force during extricating a large rigid body from the bottom of natural water reservoir (in Polish). Rozprawy Hydrotechniczne. No. 23, Instytut Budownictwa Wodnego PAN, 1969.

4. Foda M.A.: On the extrication of large objects from the ocean bottom (the breakout phenomenon). Journal of Fluid Mechanics. Vol 117, 1982.

5. Gautschi W., Slavik J.: On the Computation of Modified Bessel Function Ratios. Mathematics of Computation. Vol. 32, No. 143 (Jul., 1978), pp. 865-875.

6. Liu C.L.: Ocean sediment holding strength against breakout of imbedded objects. Tech. Rep. US Naval Civil Engineering Laboratory, Port Hueneme, California, 1969.

7. Mei C.C., Yeung R.W., Liu K.F.: Lifting of a large object from a porous seabed. Journal of Fluid Mechanics. Vol 152, 1985.

8. Mathematics.: https://math.stackexchange.com.

9. Michalski J.P.: Design method and study of autonomous hydro-location module for measuring and examining physical phenomena in marine environment (in Polish). Prace Naukowo-Badawcze WOiO, No. 01/PR/2010, Gdansk University of Technology.

10. Poinc W.: Maritime Rescue (in Polish). Wydawnictwo Morskie. Gdynia 1966. 
11. Ramy S., Ehab S., Ali H., Tasneem A.: A Simple and Efficient Approximation to the Modified Bessel Functions and Its Applications to Rician Fading. IEEE GCC Conference and exhibition, Doha, Qatar 2013.

12. Robakiewicz M.: Breakout force needed to extricate a rigid body from the bottom of a natural water reservoir (in Polish). Inżynieria Morska. No. 4/1985.

13. Sawicki A.: Soil suction forces and the breakout phenomenon. Studia Geotechnica et Mechanica. Vol. XVII, No. 1-2, 1995.

14. Sawicki A., et al.: New Type of Bottom Protection in Harbors - Design Metod. Journal of Waterway, Port, Coastal, and Ocean Engineering. July/August, 1998.

\section{CONTACT WITH THE AUTHORS}

Jan P. Michalski

e-mail:janmi@pg.edu.pl

Gdańsk University of Technology

Narutowicza 11/12

80-233 Gdansk

Poland 